TRANSACTIONS OF THE

AMERICAN MATHEMATICAL SOCIETY

Volume 352, Number 9, Pages 4019-4035

S 0002-9947(00)02559-9

Article electronically published on April 21, 2000

\title{
RESIDUES OF A PFAFF SYSTEM RELATIVE TO AN INVARIANT SUBSCHEME
}

\author{
F. SANCHO DE SALAS
}

\begin{abstract}
In this paper we give a purely algebraic construction of the theory of residues of a Pfaff system relative to an invariant subscheme. This construction is valid over an arbitrary base scheme of any characteristic.
\end{abstract}

\section{INTRODUCTION}

In 2], C. Camacho and P. Sad proved that if $\mathfrak{D}$ is a holomorphic foliation (locally, a vector field) over a complex surface and $p$ is a singular closed point of the foliation, then there exists a holomorphic curve passing through $p$ and the solution of the foliation. Their proof is based in the theorem of reduction of singularities due to Seidenberg and in the introduction of an invariant associated with the singularity and with a curve passing through it and the solution of the foliation. They called this invariant an index. Since then the notion of index has been generalized, with the name of residue, for more general situations. In the case of a singular curve of the projective plane it is due to A. Lins Neto ([7]). If the surface is a complete intersection of $\mathbb{P}^{n}(\mathbb{C})$ it is due to M. Soares $\left.(9]\right)$ and for an arbitrary complex surface to T. Suwa ([10]). In higher dimension, when the subvariety is nonsingular, it has been studied by B. Gmira ([3]) and A. Lins Neto ([] $]$ ), in the case of a hypersurface, and the general case is due to D. Lehmann ([]). Finally, in arbitrary dimension and for a singular subvariety, it is due to D. Lehmann and T. Suwa ([5], [12]). They use the differentiable structure underlying the analytic variety and the existence of connections.

In this paper we give a purely algebraic and far more general construction (valid in arbitrary characteristic) of the theory of residues of a Pfaff system $\mathcal{P}$ (without conditions of integrability), on an arbitrary $T$-scheme $X$ and relative to a closed subscheme $Y$ which is the solution of $\mathcal{P}$. The only hypothesis that we make is that the normal module of $Y$ over $X$ be a coherent locally free $\mathcal{O}_{Y}$-module. We make a technical hypothesis about the module of differentials $\Omega_{Y / T}$ : we suppose that the Koszul complex Kos. $\left(\Omega_{Y / T}\right)$ is acyclic. This is always true in characteristic 0 or

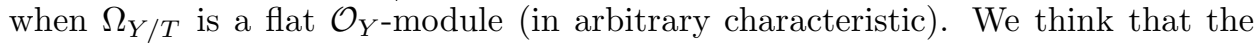
hypothesis is unnecessary, that is to say, it is always true (Conjecture 2.3).

The construction is based upon a new construction of the Atiyah extension (Theorems 1.1 and 1.2). This new construction allows one to relate the Atiyah extension

Received by the editors June 20, 1998.

2000 Mathematics Subject Classification. Primary 14B05, 14H20, 32S65; Secondary 57R20, 37C85, 57R30.

Key words and phrases. Residues, singularities, foliation. 
to the solutions of a Pfaff system (Proposition 4.1), and then to relate the Chern classes to the residues (Theorem 4.2). The local computation of the residue (under fine hypothesis) in terms of the Grothendieck symbol follows easily from the construction (Theorem 4.3). We give special attention to the case of a blowing up for its interest in desingularization. Finally we give an example of computation in the singular case.

\section{Generalized logarithmic differentials. The Atiyah extension}

Let $Y$ be a scheme over an arbitrary scheme $T$ and $N$ a locally free $\mathcal{O}_{Y}$-module.

Definition. We denote by $\mathcal{O}_{Y}[N]$ the $\mathcal{O}_{Y}$-algebra

$$
\mathcal{O}_{Y}[N]=\mathcal{O}_{Y} \oplus N
$$

with the product $(a, n) \cdot\left(a^{\prime}, n^{\prime}\right)=\left(a a^{\prime}, a n^{\prime}+a^{\prime} n\right)$; Therefore $N^{2}=0$. If we denote $\mathcal{I}$ the irrelevant ideal of $S^{\bullet} N$, then there is an isomorphism of $\mathcal{O}_{Y}$-algebras:

$$
\mathcal{O}_{Y}[N]=\left(S^{\bullet} N\right) / \mathcal{I}^{2} \text {. }
$$

Geometrically, if $Y \hookrightarrow \operatorname{Spec} S^{\bullet} N$ is the null section (which is defined by the ideal $\mathcal{I})$, then $\operatorname{Spec} \mathcal{O}_{Y}[N]$ is the first infinitesimal neighbourhood.

Let us denote $X=\operatorname{Spec} \mathcal{O}_{Y}[N]$ and $\mathcal{O}_{X}=\mathcal{O}_{Y}[N]$. One has a natural morphism $\pi: X \rightarrow Y$, which corresponds with the natural morphism of $\mathcal{O}_{Y}$-algebras $\mathcal{O}_{Y} \rightarrow$ $\mathcal{O}_{X}$, and an inmersion $Y \stackrel{j}{\hookrightarrow} X$, which corresponds with the epimorphism of $\mathcal{O}_{Y^{-}}$ algebras $\mathcal{O}_{X} \rightarrow \mathcal{O}_{Y}$, with kernel $N$.

For each $\mathcal{O}_{Y}$-module $M$, denote

$$
M_{X}=M \underset{\mathcal{O}_{Y}}{\otimes} \mathcal{O}_{X}=M \oplus\left(M \underset{\mathcal{O}_{Y}}{\otimes} N\right)
$$

Let us denote

$$
\operatorname{Der}^{N}\left(\mathcal{O}_{X}, M_{X}\right)
$$

the $\mathcal{O}_{X}$-module of the derivations (over $T$ ) of $\mathcal{O}_{X}$ in $M_{X}$ that leave $N$ stable (that is, that take $N$ into $\left.N \cdot M_{X}=M \otimes N\right)$.

Proposition 1.1. One has an exact and locally split sequence:

$$
0 \rightarrow \operatorname{Hom}_{Y}\left(N, M \underset{\mathcal{O}_{Y}}{\otimes} N\right) \rightarrow \operatorname{Der}^{N}\left(\mathcal{O}_{X}, M_{X}\right) \underset{\mathcal{O}_{X}}{\otimes} \mathcal{O}_{Y} \rightarrow \operatorname{Der}\left(\mathcal{O}_{Y}, M\right) \rightarrow 0
$$

which is functorial in $M$.

Proof. If $D: \mathcal{O}_{X} \rightarrow M_{X}$ is a derivation that leaves $N$ stable, then it induces a derivation in the quotient $\mathcal{O}_{Y}=\mathcal{O}_{X} / N \rightarrow M_{X} / N=M$. That is, one has a morphism

$$
\operatorname{Der}^{N}\left(\mathcal{O}_{X}, M_{X}\right) \rightarrow \operatorname{Der}\left(\mathcal{O}_{Y}, M\right)
$$

and then a morphism

$$
\phi: \operatorname{Der}^{N}\left(\mathcal{O}_{X}, M_{X}\right) \underset{\mathcal{O}_{X}}{\otimes} \mathcal{O}_{Y} \rightarrow \operatorname{Der}\left(\mathcal{O}_{Y}, M\right)
$$

On the other hand, a morphism $f: N \rightarrow M \otimes N$ induces a derivation $D_{f}: \mathcal{O}_{X} \rightarrow$ $M_{X}$, defined by $D_{f}(a, n)=(0, f(n))$, that leaves $N$ stable. Then we have a morphism

$$
\operatorname{Hom}_{Y}\left(N, M \underset{\mathcal{O}_{Y}}{\otimes} N\right) \rightarrow \operatorname{Der}^{N}\left(\mathcal{O}_{X}, M_{X}\right)
$$


and then a morphism

$$
i: \operatorname{Hom}_{Y}\left(N, M \underset{\mathcal{O}_{Y}}{\otimes} N\right) \rightarrow \operatorname{Der}^{N}\left(\mathcal{O}_{X}, M_{X}\right) \underset{\mathcal{O}_{X}}{\otimes} \mathcal{O}_{Y}
$$

The exactness and local triviality of the sequence follows if $N$ is a free module and $e_{1}, \ldots, e_{n}$ is a basis, then we may define a morphism

$$
\operatorname{Der}\left(\mathcal{O}_{Y}, M\right) \rightarrow \operatorname{Der}^{N}\left(\mathcal{O}_{X}, M_{X}\right)
$$

in the following way: given a derivation $D: \mathcal{O}_{Y} \rightarrow M$, we define $\widetilde{D}: \mathcal{O}_{X} \rightarrow M_{X}$ by $\widetilde{D}\left(a, \sum a_{i} e_{i}\right)=\left(D a, \sum\left(D a_{i}\right) \otimes e_{i}\right)$, that is, $\widetilde{D} e_{i}=0$. It is easy to check that $\widetilde{D}$ is a derivation that leaves $N$ stable. We have then a morphism

$$
s: \operatorname{Der}\left(\mathcal{O}_{Y}, M\right) \rightarrow \operatorname{Der}^{N}\left(\mathcal{O}_{X}, M_{X}\right) \underset{\mathcal{O}_{X}}{\otimes} \mathcal{O}_{Y}
$$

and a straightforward verification shows that the composition $s \circ \phi$ is the identity. Moreover, it is easy to see that if $D \in \operatorname{Der}^{N}\left(\mathcal{O}_{X}, M_{X}\right) \underset{\mathcal{O}_{X}}{\otimes} \mathcal{O}_{Y}$, then $D-(s \circ \phi)(D)$ is a morphism of $N$ in $M \otimes N$. Conclusion follows.

Theorem 1.2 (logarithmic differentials). The functor

$$
F(M)=\operatorname{Der}^{N}\left(\mathcal{O}_{X}, M_{X}\right) \underset{\mathcal{O}_{X}}{\otimes} \mathcal{O}_{Y}
$$

is representable. The representant will be denoted by $\Omega_{N}[Y]$ and will be called logarithmic differentials of $N$. One has an extension

$$
0 \rightarrow \Omega_{Y / T} \rightarrow \Omega_{N}[Y] \rightarrow\left(E n d_{Y} N\right)^{*} \rightarrow 0 .
$$

Proof. The natural morphism $\left(\operatorname{End}_{Y} N\right) \underset{\mathcal{O}_{Y}}{\otimes} M \rightarrow \operatorname{Hom}_{Y}\left(N, M \underset{\mathcal{O}_{Y}}{\otimes} N\right)$ is an isomorphism, so the functor in $M, \operatorname{Hom}_{Y}\left(N, M \underset{\mathcal{O}_{Y}}{\otimes} N\right)$, is representable by $\left(\operatorname{End}_{Y} N\right)^{*}$. On the other hand, the functor $\operatorname{Der}_{T}\left(\mathcal{O}_{Y}, M\right)$ is representable by the module of differentials, $\Omega_{Y / T}$. One concludes by the Proposition 1.1 before.

Remark. If $N$ is the conormal module of a hypersurface $Y$ of a smooth variety $X$, then $\Omega_{N}[Y]=\Omega_{X}[\log Y] \underset{\mathcal{O}_{X}}{\otimes} \mathcal{O}_{Y}$. That is why we call $\Omega_{N}[Y]$ logarithmic differentials.

Theorem 1.3. The extension $0 \rightarrow \Omega_{Y / T} \rightarrow \Omega_{N}[Y] \rightarrow\left(E_{n d} N\right)^{*} \rightarrow 0$ is the Atiyah extension ([1]).

Proof. It is enough to see that the splittings of the extension (1.2) correspond with the connections over $N$. Let us denote $T_{Y}=\operatorname{Der}\left(\mathcal{O}_{Y}, \mathcal{O}_{Y}\right)=\Omega_{Y / T}^{*}$, and $\mathcal{O}_{X}=\mathcal{O}_{Y} \oplus N$. Given a connection over $N$, one has a morphism $\nabla: T_{Y} \rightarrow$ $\operatorname{End}_{T}(N)$. Then one has a morphism $T_{Y} \rightarrow \operatorname{Der}^{N}\left(\mathcal{O}_{X}, \mathcal{O}_{X}\right)$ defined as $D \mapsto \widetilde{D}$, $\widetilde{D}(a, n)=(D a, \nabla(D)(n))$, and then a morphism

$$
T_{Y} \rightarrow \operatorname{Der}^{N}\left(\mathcal{O}_{X}, \mathcal{O}_{X}\right) \underset{\mathcal{O}_{X}}{\otimes} \mathcal{O}_{Y}=\left(\Omega_{N}[Y]\right)^{*}
$$

and taking dual, $\Omega_{N}[Y] \rightarrow \Omega_{Y / T}$, which is a section of $\Omega_{Y / T} \rightarrow \Omega_{N}[Y]$.

Conversely, given a splitting of $0 \rightarrow \Omega_{Y / T} \rightarrow \Omega_{N}[Y] \rightarrow\left(\operatorname{End}_{Y} N\right)^{*} \rightarrow 0$, one has a morphism $\Omega_{N}[Y] \rightarrow \Omega_{Y / T}$, and taking dual $T_{Y} \rightarrow \operatorname{Der}^{N}\left(\mathcal{O}_{X}, \mathcal{O}_{X}\right) \underset{\mathcal{O}_{X}}{\otimes} \mathcal{O}_{Y}$. Now, 
there is a natural morphism $\operatorname{Der}^{N}\left(\mathcal{O}_{X}, \mathcal{O}_{X}\right) \rightarrow \operatorname{End}_{T}(N)$ and then a morphism $\operatorname{Der}^{N}\left(\mathcal{O}_{X}, \mathcal{O}_{X}\right) \underset{\mathcal{O}_{X}}{\otimes} \mathcal{O}_{Y} \rightarrow \operatorname{End}_{T}(N)$. The composition

$$
T_{Y} \rightarrow \operatorname{Der}^{N}\left(\mathcal{O}_{X}, \mathcal{O}_{X}\right) \underset{\mathcal{O}_{X}}{\otimes} \mathcal{O}_{Y} \rightarrow \operatorname{End}_{T}(N)
$$

is the desired connection.

\section{Koszul complex}

The results of this section are well known. They are stated without proofs.

Let $\mathcal{O}$ be a sheaf of rings over a scheme $Y, M$ an $\mathcal{O}$-module and $S^{\bullet} M$ its symmetric algebra.

Proposition. One has an isomorphism of $S^{\bullet} M$-modules

$$
\Omega_{S} \bullet M / \mathcal{O}=M \underset{\mathcal{O}}{\otimes} S^{\bullet} M
$$

and therefore $\Omega_{S \cdot M / \mathcal{O}}^{k}=\Lambda^{k} M \underset{\mathcal{O}}{\otimes} S^{\bullet} M$

The natural morphism $M \rightarrow S^{\bullet} M$ induces a derivation over $\mathcal{O}$

$$
D: S^{\bullet} M \rightarrow S^{\bullet} M
$$

which we call universal derivation of $M$. If $S^{\bullet} M=\mathcal{O}\left[\xi_{1}, \ldots, \xi_{n}\right]$, then $D=\sum_{i} \xi_{i} \frac{\partial}{\partial \xi_{i}}$.

Definition. The inner contraction with $D$ defines a complex



which we shall call the universal Koszul complex of $M$. In conclusion,

$$
\operatorname{Kos}_{\bullet}(M)=\Omega_{S \bullet M / \mathcal{O}}^{\bullet}=\Lambda^{\bullet} M \underset{\mathcal{O}}{\otimes} S^{\bullet} M
$$

and the differential is the inner contraction with $D$.

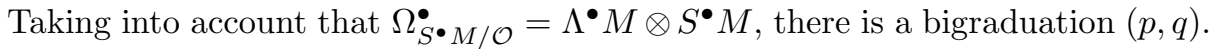
With regard to it, $i_{D}$ has degree $(-1,1)$. If we take the induced simple graduation (that is, $\Lambda^{p} M \otimes S^{q} M$ has degree $p+q$ ), then $i_{D}$ is homogeneous of degree zero.

Definition. We shall denote $\operatorname{Kos}_{\bullet}^{p}(M)$ the homogeneous component of degree $p$ of the complex $\operatorname{Kos}_{\bullet}(M)$, considering the simple graduation associated with the bigraduation $(p, q)$. That is, $\operatorname{Kos}_{\bullet}^{p}(M)$ is the complex

$$
\begin{aligned}
0 \rightarrow \Lambda^{p} M & \stackrel{i_{D}}{\rightarrow} \Lambda^{p-1} M \otimes M \stackrel{i_{D}}{\rightarrow} \cdots \\
& \rightarrow \Lambda^{p-i} M \otimes S^{i} M \stackrel{i_{D}}{\rightarrow} \cdots \rightarrow M \otimes S^{p-1} M \stackrel{i_{D}}{\rightarrow} S^{p} M \rightarrow 0
\end{aligned}
$$

and one has that $\operatorname{Kos}_{\bullet}(M)=\bigoplus_{p} \operatorname{Kos}_{\bullet}^{p}(M)$.

Theorem 2.1. If $\mathcal{O}$ is an algebra over a field $k$ of characteristic zero, then the complex $\operatorname{Kos}_{\bullet}(M)$ is acyclic (that is, it is a resolution of $\mathcal{O}$ ). In characteristic $p>0$, if $n$ and $p$ are relatively prime, then $\operatorname{Kos}_{\bullet}^{n}(M)$ is acyclic.

Theorem 2.2. If $M$ is flat, then the universal Koszul complex of $M$ is acyclic.

Conjecture 2.3. The Koszul complex $\operatorname{Kos}_{\bullet}(M)$ is always acyclic (for an arbitrary module $M$, in arbitrary characteristic). 
Let $f: M \rightarrow N$ be a morphism of $\mathcal{O}$-modules, which induces a morphism of $\mathcal{O}$-algebras $S^{\bullet} f: S^{\bullet} M \rightarrow S^{\bullet} N$.

Definition. We shall call the Koszul complex of $f, \operatorname{Kos} \bullet(f)$, to the complex

$$
\operatorname{Kos}_{\bullet}(f)=\operatorname{Kos}_{\bullet}(M) \underset{S^{\bullet} M}{\otimes} S^{\bullet} N=\Lambda^{\bullet} M \underset{\mathcal{O}}{\otimes} S^{\bullet} N
$$

where the differential is the one induced by the differential of $\operatorname{Kos}_{\bullet}(M)$. Equivalently, if we denote $D_{f}: S^{\bullet} M \rightarrow S^{\bullet} N$ the derivation which is the composition of $S^{\bullet} f$ with the universal derivation of $M$, then Kos• $(f)$ is the complex

$$
\operatorname{Kos}_{\bullet}(f)=\Omega_{S \bullet M / \mathcal{O}}^{\bullet} \underset{S \bullet M}{\otimes} S^{\bullet} N
$$

and the differential is the inner contraction with $D_{f}$.

By definition one has that

$$
\operatorname{Kos}_{\bullet}(I d)=\operatorname{Kos}_{\bullet}(M)
$$

where $I d: M \rightarrow M$ is the identity morphism.

Analogously, one defines $\operatorname{Kos}_{\bullet}^{p}(f)$ as the homogeneous component of degree $p$ of the complex $\operatorname{Kos}_{\bullet}(f)$, by considering the simple graduation associated with the bigraduation $\Lambda^{p} M \otimes S^{q} N$. That is, $\operatorname{Kos}_{\bullet}^{p}(f)$ is the complex

$$
\begin{aligned}
0 \rightarrow & \Lambda^{p} M \stackrel{i_{D_{f}}}{\rightarrow} \Lambda^{p-1} M \otimes N \stackrel{i_{D_{f}}}{\rightarrow} \\
& \quad \cdots \rightarrow \Lambda^{p-i} M \otimes S^{i} N \stackrel{i_{D_{f}}}{\rightarrow} \cdots \rightarrow M \otimes S^{p-1} N \stackrel{i_{D_{f}}}{\rightarrow} S^{p} N \rightarrow 0 .
\end{aligned}
$$

Functoriality: Given a commutative diagram of modules and morphism of modules

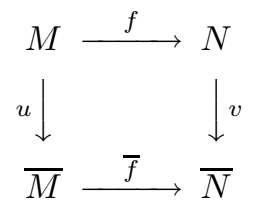

it induces a morphism of complexes $\operatorname{Kos}_{\bullet}(f) \rightarrow \operatorname{Kos}_{\bullet}(\bar{f})$. Analogously, for each $p$, one has a morphism of complexes $\operatorname{Kos}_{\bullet}^{p}(f) \rightarrow \operatorname{Kos}_{\bullet}^{p}(\bar{f})$.

Proposition 2.4. Let $0 \rightarrow M_{1} \stackrel{f}{\rightarrow} M_{2} \rightarrow M_{3} \rightarrow 0$ be an exact sequence of modules. Suppose that $M_{3}$ is flat and that $\operatorname{Kos}_{\bullet}\left(M_{1}\right)$ is acyclic. Then $\operatorname{Kos}_{\bullet}(f)$ is a resolution of $S^{\bullet} M_{3}$. Analogously $\operatorname{Kos}_{\bullet}^{p}(f)$ is a resolution of $S^{p} M_{3}$.

If $\operatorname{Kos}_{\bullet}^{p}(f)$ is a resolution of $S^{p} M_{3}$ of length $p$, it induces a morphism in cohomology $H^{0}\left(Y, S^{p} M_{3}\right) \rightarrow H^{p}\left(Y, \Lambda^{p} M_{1}\right)$. Then we have a morphism of graded $H^{0}(Y, \mathcal{O})$-algebras

$$
\theta_{f}: H^{0}\left(Y, S^{\bullet} M_{3}\right) \rightarrow \bigoplus_{p \geq 0} H^{p}\left(Y, \Lambda^{p} M_{1}\right)
$$

where the second member is an algebra with the cup product associated with the exterior product. 


\section{ChERN CLASSES}

Let us denote $\Omega_{Y / T}^{p}=\Lambda^{p} \Omega_{Y / T}$. In this section we are always going to suppose that $\operatorname{Kos}_{\bullet}\left(\Omega_{Y / T}\right)$ is acyclic. This is always true in characteristic zero, and in arbitrary characteristic if $\Omega_{Y / T}$ is flat (see Theorems 2.1 and 2.2). This hypothesis is superfluous if Conjecture 2.3 is true.

For simplicity we shall denote $\Omega_{Y}^{p}=\Omega_{Y / T}^{p}$. Let us consider the (Hodge) cohomology ring

$$
A^{\bullet}(Y)=\bigoplus_{p \geq 0} H^{p}\left(Y, \Omega_{Y}^{p}\right)
$$

with the graduation $A^{p}(Y)=H^{p}\left(Y, \Omega_{Y}^{p}\right)$, and the cup product associated with the exterior product.

Let $N$ be a locally free module over $Y$. The Atiyah extension $0 \rightarrow \Omega_{Y / T} \rightarrow$ $\Omega_{N}[Y] \rightarrow\left(\operatorname{End}_{Y} N\right)^{*} \rightarrow 0$ induces, by the Koszul complex (see (2.5)) a morphism of graded algebras

$$
\theta_{N}: H^{0}\left(Y, S^{\bullet}\left(\operatorname{End}_{Y} N\right)^{*}\right) \longrightarrow A^{\bullet}(Y)
$$

that is, for each $p$ a morphism $H^{0}\left(Y, S^{p}\left(\operatorname{End}_{Y} N\right)^{*}\right) \longrightarrow A^{p}(Y)$.

Theorem 3.1 ([1, p. 197], [4, pp. 295-296]). If $s_{j} \in H^{0}\left(Y, S^{\bullet}\left(\operatorname{End}_{Y} N\right)^{*}\right)$ is the function that assigns, to each endomorphism, the $j$-th coefficient of its characteristic polynomial $\left(s_{1}=-\right.$ trace, $\ldots, s_{r}=(-1)^{r}$ det. $)$, then

$$
\theta_{N}\left(s_{j}\right)=c_{j}(N)
$$

where $c_{j}(N)$ is the $j$-th chern class of $N$ valued at $A^{\bullet}(Y)$.

\section{Residues of a Pfaff System Relative to AN INVARiant subscheme}

Let $X$ be a $T$-scheme. Let us denote $\Omega_{X}$ the module of differentials of $X$ over $T$.

Definition. A Pfaff system of rank $r$ on $X$ (over $T$ ) is a morphism of $\mathcal{O}_{X}$-modules

$$
\Omega_{X} \rightarrow \mathcal{P}
$$

where $\mathcal{P}$ is a locally free module of rank $r$. Equivalently, it is a derivation $D: \mathcal{O}_{X} \rightarrow$ $\mathcal{P}$. Usually we shall simply say that $\mathcal{P}$ is a Pfaff system on $X$.

If $\mathrm{P}=\operatorname{Spec} S^{\bullet} \mathcal{P}^{*}$, and $\mathbb{T}_{X}=\operatorname{Spec} S^{\bullet} \Omega_{X}$ is the tangent "bundle", this is equivalent to giving a morphism

$$
\mathrm{P} \rightarrow \mathbb{T}_{X}
$$

In the context of analytic geometry, this would be a holomorphic foliation of dimension $r$, parametrized by $T$.

Definition. The singular locus of a Pfaff system $\mathcal{P}$, is the set of points $p \in X$ such that the morphism

$$
\Omega_{X} \otimes k(p) \rightarrow \mathcal{P} \otimes k(p)
$$

is not an epimorphism, where $k(p)$ denotes the residual field of $p$. We shall denote it by $\operatorname{Sin}(\mathcal{P})$. 
Equivalently, it is the set of points where the morphism $\mathrm{P} \rightarrow \mathbb{T}_{X}$ is not injective. If $C$ is the cokernel of $\Omega_{X} \rightarrow \mathcal{P}$, then the singular locus of $\mathcal{P}$ is the support of $C$. So we shall give it a scheme structure by putting

$$
\operatorname{Sin}(\mathcal{P})=\operatorname{Spec} \mathcal{O}_{X} / F_{0}(C)
$$

where $F_{0}(C)$ is the 0 -th Fitting ideal of $\mathcal{P}$.

For each point $x \in X$, let us denote $\mathcal{O}_{x}$ the local ring at $x$ and $k(x)$ the residual field of $x$. Let us denote $m_{x}=\operatorname{dim}_{k(x)} \Omega_{X, x} \underset{\mathcal{O}_{x}}{\otimes} k(x)$ and

$$
d(X)=\min _{x \in X} m_{x}
$$

If $X$ is irreducible, then $d(X)=m_{g}$, where $g$ is the generic point of $X$. If $X \rightarrow T$ is smooth, then $d(X)$ is the relative dimension of $X \rightarrow T$.

Definition. We shall denote by $\operatorname{Sin}(X)$ the set of points $x \in X$ such that $m_{x}>$ $d(X)$. It is a closed subset; indeed, it is the support of $\Lambda^{1+d(X)} \Omega_{X}$.

Definition. Let $\mathcal{P}$ be a Pfaff system on $X$ and $j: Y \hookrightarrow X$ a closed subscheme. We say that $Y$ is the solution of $\mathcal{P}$, or that $Y$ is invariant by $\mathcal{P}$, if the morphism

$$
j^{*} \Omega_{X} \rightarrow j^{*} \mathcal{P}
$$

factors through $\Omega_{Y}$. If $Y$ is the solution of $\mathcal{P}$, then $\mathcal{P}_{\mid Y}=j^{*} \mathcal{P}$ is a Pfaff system on $Y$.

In terms of the derivation $D: \mathcal{O}_{X} \rightarrow \mathcal{P}, Y$ is the solution of $\mathcal{P}$ if and only if $D \mathfrak{p} \subseteq \mathfrak{p} \mathcal{P}$, where $\mathfrak{p}$ is the sheaf of ideals of $Y$.

The main fact is the following:

Proposition 4.1. Let $Y$ be a solution of a Pfaff system $\mathcal{P}$ on $X$, and let us denote $N=\mathfrak{p} / \mathfrak{p}^{2}$ the conormal module of $Y$ over $X$. Suppose that $N$ is a locally free $\mathcal{O}_{Y}$-module. Then there exists a morphism

$$
\Omega_{N}[Y] \longrightarrow \mathcal{P}_{\mid Y}
$$

such that

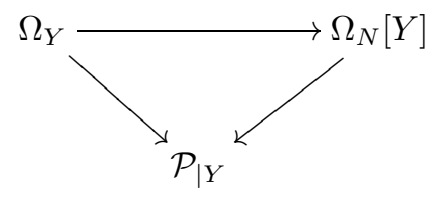

is commutative.

Proof. Let $D: \mathcal{O}_{X} \rightarrow \mathcal{P}$ be the derivation associated with the Pfaff system. Since $Y$ is the solution of $\mathcal{P}$, one has that $D \mathfrak{p} \subseteq \mathfrak{p} \mathcal{P}$, so it induces a derivation

$$
\mathcal{O}_{Y}=\mathcal{O}_{X} / \mathfrak{p} \rightarrow \mathcal{P} / \mathfrak{p} \mathcal{P}=\mathcal{P}_{\mid Y}
$$

and another

$$
\mathfrak{p} / \mathfrak{p}^{2} \rightarrow \mathfrak{p} \mathcal{P} / \mathfrak{p}^{2} \mathcal{P}=\mathfrak{p} / \mathfrak{p}^{2} \underset{\mathcal{O}_{Y}}{\otimes} \mathcal{P}_{\mid Y}
$$

so, taking the direct sum, one has a derivation

$$
\mathcal{O}_{Y}[N] \rightarrow \mathcal{P}_{\mid Y}[N]
$$

that corresponds with a morphism $\Omega_{N}[Y] \rightarrow \mathcal{P}_{\mid Y}$. The commutativity of the diagram is immediate. 
Theorem 4.2 (existence of residue). Let $\mathcal{P}$ be a Pfaff system of rank $r$ on $X$. Let $Y \stackrel{j}{\hookrightarrow} X$ be a closed subscheme defined by the sheaf of ideals $\mathfrak{p}$ and solution of $\mathcal{P}$. Suppose that $\mathfrak{p} / \mathfrak{p}^{2}$ is a locally free module and denote $S=\operatorname{Sin}\left(\mathcal{P}_{\mid Y}\right) \cup \operatorname{Sin}(Y)$. If $p>d(Y)-r$, then the morphism (see (3.0))

$$
\theta_{p}: H^{0}\left(Y, S^{p} \operatorname{End}_{Y}\left(\mathfrak{p} / \mathfrak{p}^{2}\right)^{*}\right) \longrightarrow H^{p}\left(Y, \Omega_{Y}^{p}\right)
$$

factors through $H_{S}^{p}\left(Y, \Omega_{Y}^{p}\right)$.

Proof. Let us denote $N=\mathfrak{p} / \mathfrak{p}^{2}$ and

$$
\begin{aligned}
K^{\prime} & =\text { kernel of } \Omega_{Y} \rightarrow \mathcal{P}_{\mid Y} \\
K & =\text { kernel of } \Omega_{N}[Y] \rightarrow \mathcal{P}_{\mid Y} \\
\bar{K} & =\text { image of } K \text { in } \operatorname{End} d_{Y}\left(\mathfrak{p} / \mathfrak{p}^{2}\right)^{*}
\end{aligned}
$$

One has a commutative diagram

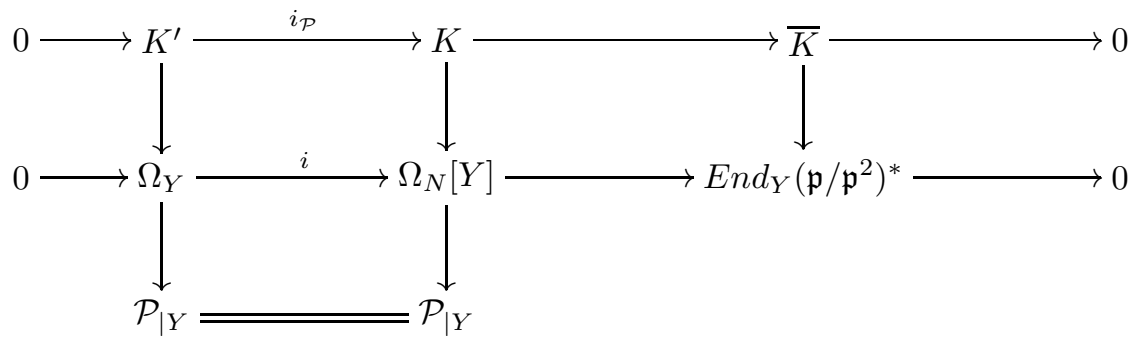

that induces a morphism of complexes

$$
\operatorname{Kos}_{\bullet}\left(i_{\mathcal{P}}\right) \rightarrow \operatorname{Kos}_{\bullet}(i)
$$

Let $U=Y-S$. Restricting to $U$, the morphisms $\Omega_{Y} \rightarrow \mathcal{P}_{\mid Y}$ and $\Omega_{N}[Y] \rightarrow \mathcal{P}_{\mid Y}$ are epimorphisms, so $K^{\prime}$ and $K$ are, over $U$, locally direct summands of $\Omega_{Y}$ and $\Omega_{N}[Y]$ respectively, and the quotient $\bar{K}$ is isomorphic to $\operatorname{End} d_{Y}\left(\mathfrak{p} / \mathfrak{p}^{2}\right)^{*}$ over $U$. Therefore $\mathrm{Kos}_{\bullet}\left(i_{\mathcal{P}}\right)$ is a resolution of $S^{\bullet} \bar{K}$ on $U$.

Given a sheaf $F$ on $Y$, let us denote $F_{U}$ (resp. $F_{S}$ ) the sheaf supported on $U$ (resp. on $S$ ). One has an exact sequence $0 \rightarrow F_{U} \rightarrow F \rightarrow F_{S} \rightarrow 0$.

By the discussion above, one has that $\operatorname{Kos}_{\bullet}\left(i_{\mathcal{P}}\right)_{U}$ is a resolution of the sheaf $\left(S^{\bullet} \bar{K}\right)_{U}=\left(S^{\bullet} \operatorname{End} d_{Y}\left(\mathfrak{p} / \mathfrak{p}^{2}\right)^{*}\right)_{U}$ and one has an inclusion

$$
\operatorname{Kos}_{\bullet}\left(i_{\mathcal{P}}\right)_{U} \hookrightarrow \operatorname{Kos}_{\bullet}(i)
$$

Let $K_{\bullet}$ be the quotient complex $\operatorname{Kos}_{\bullet}(i) / \operatorname{Kos}_{\bullet}\left(i_{\mathcal{P}}\right)_{U}$, which is a resolution of the sheaf $\left(S^{\bullet} \operatorname{End}_{Y}\left(\mathfrak{p} / \mathfrak{p}^{2}\right)^{*}\right)_{S}$. Analogously, for each $p, K_{\bullet}^{p}=\operatorname{Kos}_{\bullet}^{p}(i) / \operatorname{Kos}_{\bullet}^{p}\left(i_{\mathcal{P}}\right)_{U}$ is a resolution of $\left(S^{p} \operatorname{End}_{Y}\left(\mathfrak{p} / \mathfrak{p}^{2}\right)^{*}\right)_{S}$. If $p$ is greater than $d(Y)-r$, then $\left(\Lambda^{p} K^{\prime}\right)_{U}=0$, because $K^{\prime}$ is, over $U$, a locally direct summand of $\Omega_{Y}$. Therefore, if $p>d(Y)-r$, then $K_{\bullet}^{p}$ is an extension of $\left(S^{p} E n d_{Y}\left(\mathfrak{p} / \mathfrak{p}^{2}\right)^{*}\right)_{S}$ by $\Omega_{Y}^{p}$, and the natural morphism 
$\operatorname{Kos}_{\bullet}^{p}(i) \rightarrow K_{\bullet}^{p}$ induces a commutative diagram

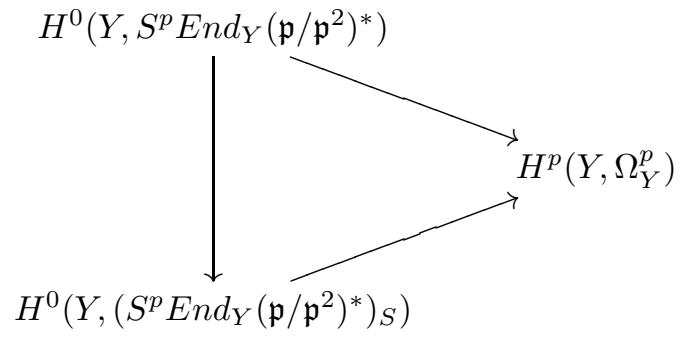

Now,

$$
H^{0}\left(Y,\left(S^{p} \operatorname{End}_{Y}\left(\mathfrak{p} / \mathfrak{p}^{2}\right)^{*}\right)_{S}\right)=H_{S}^{0}\left(Y,\left(S^{p} \operatorname{End}_{Y}\left(\mathfrak{p} / \mathfrak{p}^{2}\right)^{*}\right)_{S}\right)
$$

and hence the morphism $H_{S}^{0}\left(Y,\left(S^{p} E n d_{Y}\left(\mathfrak{p} / \mathfrak{p}^{2}\right)^{*}\right)_{S}\right) \rightarrow H^{p}\left(Y, \Omega_{Y}^{p}\right)$ factors through $H_{S}^{p}\left(Y, \Omega_{Y}^{p}\right)$, so the conclusion follows.

Remark. Let $d$ be the minimum integer such that $\Lambda^{d} \Omega_{Y}=0$. The proof of the theorem shows that if $p \geq d-r$, then the residues are localized not only in $S=$ $\operatorname{Sin}\left(\mathcal{P}_{\mid Y}\right) \cup \operatorname{Sin}(Y)$ but in $\operatorname{Sin}\left(\mathcal{P}_{\mid Y}\right)$.

Definition. Let $Q$ be a homogeneous polynomial of $l\left(=\operatorname{rank}\right.$ of $\left.N_{Y / X}\right)$ variables and degree $p>d(Y)-r$ (assume that the variable $x_{i}$ has degree $i$ ). If $S_{\alpha}$ are the connected components of $S=\operatorname{Sin}\left(\mathcal{P}_{\mid Y}\right) \cup \operatorname{Sin}(Y)$, we shall denote by $\operatorname{Res}_{S_{\alpha}}(\mathcal{P}, Q)$ the image of $Q\left(s_{1}, \ldots, s_{l}\right)$ in $H_{S_{\alpha}}^{p}\left(Y, \Omega_{Y}^{p}\right)$ under the morphism

$$
H^{0}\left(Y, S^{p} \operatorname{End}_{Y}\left(\mathfrak{p} / \mathfrak{p}^{2}\right)^{*}\right) \rightarrow H_{S}^{p}\left(Y, \Omega_{Y}^{p}\right)=\bigoplus_{\alpha} H_{S_{\alpha}}^{p}\left(Y, \Omega_{Y}^{p}\right) \rightarrow H_{S_{\alpha}}^{p}\left(Y, \Omega_{Y}^{p}\right) .
$$

By construction and Theorem 3.1, one has that

$$
\sum_{\alpha} \phi_{\alpha}\left(\operatorname{Res}_{S_{\alpha}}(\mathcal{P}, Q)\right)=Q\left(c_{1}\left(\mathfrak{p} / \mathfrak{p}^{2}\right), \ldots, c_{l}\left(\mathfrak{p} / \mathfrak{p}^{2}\right)\right)
$$

where $\phi_{\alpha}: H_{S_{\alpha}}^{p}\left(Y, \Omega_{Y}^{p}\right) \rightarrow H^{p}\left(Y, \Omega_{Y}^{p}\right)$ is the natural morphism and $c_{i}\left(\mathfrak{p} / \mathfrak{p}^{2}\right)$ is $i$-th Chern class of $\mathfrak{p} / \mathfrak{p}^{2}$ with values in the Hodge cohomology.

Remarks. 1) In the complex analytic case the residues are usually expressed as localizations of the characteristic classes of the normal module $\mathfrak{p} / \mathfrak{p}^{2^{*}}$. Taking into account that $c_{i}\left(\mathfrak{p} / \mathfrak{p}^{2}\right)=(-1)^{i} c_{i}\left(\mathfrak{p} / \mathfrak{p}^{2^{*}}\right)$, one can obtain the definition given in the literature for the complex analytic case from the one given here.

2) The proof of the latter theorem shows why the integrability of the Pfaff system is not neccesary. It is only neccesary that, out of the singular locus, the kernel of the morphism $\Omega_{Y} \rightarrow \mathcal{P}_{\mid Y}$ is a locally free module of rank $d(Y)-r$.

Explicit computation. Hypothesis: Suppose that $Y$ is smooth. Then $S=$ $\operatorname{Sin}\left(\mathcal{P}_{\mid Y}\right)$. Assume that $S \hookrightarrow Y$ is a locally complete intersection of codimension $d$. Then

$$
H_{S}^{d}\left(Y, \Omega_{Y}^{d}\right)=H^{0}\left(S, \mathcal{H}_{S}^{d}\left(Y, \Omega_{Y}^{d}\right)\right)
$$

and therefore the residue may be computed locally. 
Grothendieck symbol. Let $f_{1}, \ldots, f_{d} \in H^{0}\left(Y, \mathcal{O}_{Y}\right)$ be a regular sequence and $w \in H^{0}\left(Y, \Omega_{Y}^{d}\right)$. We are going to define an element

$$
\left[\begin{array}{c}
\omega \\
f_{1}, \ldots, f_{d}
\end{array}\right] \in \operatorname{Ext}_{\mathcal{O}_{Y}}^{d}\left(\mathcal{O}_{Y} /\left(f_{1}, \ldots, f_{d}\right), \Omega_{Y}^{d}\right)
$$

which is called the Grothendieck symbol.

Since $f_{1}, \ldots, f_{d}$ is a regular sequence, the Koszul complex $\left(\mathcal{O}\right.$ stands for $\left.\mathcal{O}_{Y}\right)$

$$
0 \rightarrow \Lambda^{d} L \rightarrow \cdots \rightarrow L \rightarrow \mathcal{O} \rightarrow \mathcal{O} /\left(f_{1}, \ldots, f_{r}\right) \rightarrow 0, \quad L=\mathcal{O} \oplus \stackrel{d}{ } \rightarrow \oplus \mathcal{O}
$$

is acyclic; hence, it gives an element $\operatorname{Kos}_{\bullet}\left(f_{1}, \ldots, f_{d}\right) \in \operatorname{Ext}_{\mathcal{O}}^{d}\left(\mathcal{O} /\left(f_{1}, \ldots, f_{d}\right), \Lambda^{d} L\right)$.

Moreover $w$ defines a morphism $\Lambda^{d} L \rightarrow \Omega_{Y}^{d}$, that maps $e_{1} \wedge \cdots \wedge e_{d}$ into $w$, and then a morphism.

$$
\operatorname{Ext}_{\mathcal{O}}^{d}\left(\mathcal{O} /\left(f_{1}, \ldots, f_{d}\right), \Lambda^{d} L\right) \rightarrow \operatorname{Ext}_{\mathcal{O}}^{d}\left(\mathcal{O} /\left(f_{1}, \ldots, f_{d}\right), \Omega_{Y}^{d}\right) .
$$

Then $\left[\begin{array}{c}\omega \\ f_{1}, \ldots, f_{d}\end{array}\right] \in \operatorname{Ext}_{\mathcal{O}_{Y}}^{d}\left(\mathcal{O}_{Y} /\left(f_{1}, \ldots, f_{d}\right), \Omega_{Y}^{d}\right)$ is the image of $\operatorname{Kos}_{\bullet}\left(f_{1}, \ldots, f_{d}\right)$ by the latter morphism.

Denote $S=\left(f_{1}, \ldots, f_{d}\right)_{0}$. Taking into account that $\operatorname{Ext}_{\mathcal{O}}^{d}\left(\mathcal{O} /\left(f_{1}, \ldots, f_{d}\right), \Omega_{Y}^{d}\right) \hookrightarrow$ $H_{S}^{d}\left(Y, \Omega_{Y}^{d}\right)$, the Grothendieck symbol $\left[\begin{array}{c}\omega \\ f_{1}, \ldots, f_{d}\end{array}\right]$, considered in $H_{S}^{d}\left(Y, \Omega_{Y}^{d}\right)$, is the image of 1 by the morphisms

$$
H^{0}\left(S, \mathcal{O} /\left(f_{1}, \ldots, f_{d}\right)\right) \rightarrow H_{S}^{d}\left(Y, \Lambda^{d} L\right) \rightarrow H_{S}^{d}\left(Y, \Omega_{Y}^{d}\right)
$$

where $H^{0}\left(S, \mathcal{O} /\left(f_{1}, \ldots, f_{d}\right)\right) \rightarrow H_{S}^{d}\left(Y, \Lambda^{d} L\right)$ is the morphism induced by the Koszul complex $0 \rightarrow \Lambda^{d} L \rightarrow \cdots \rightarrow L \rightarrow \mathcal{O} \rightarrow \mathcal{O} /\left(f_{1}, \ldots, f_{r}\right) \rightarrow 0$ and $H_{S}^{d}\left(Y, \Lambda^{d} L\right) \rightarrow$ $H_{S}^{d}\left(Y, \Omega_{Y}^{d}\right)$ is the one induced by $\Lambda^{d} L \rightarrow \Omega_{Y}^{d}$.

Local structure of the Pfaff system. Let $C$ be the cokernel of $\Omega_{Y} \rightarrow \mathcal{P}_{\mid Y}$. Let us consider the chain of closed subschemes

$$
S^{r-1} \subset \cdots \subset S^{2} \subset S^{1}=S
$$

defined by $S^{i}=\left\{y \in Y\right.$ such that $\left.\operatorname{dim}_{k(y)} C_{y} \otimes k(y) \geq i\right\}$. $S^{i}$ is the closed subscheme defined by the Fitting ideal $F_{i}(C)$. If $y \in S-S^{2}$, then, by Nakayama's lemma, there exist

$$
\begin{gathered}
\omega_{1}, \ldots, \omega_{n} \text { basis of } \Omega_{Y, y}, \\
D_{1}, \ldots, D_{r} \text { basis of } \mathcal{P}_{\mid Y, y}
\end{gathered}
$$

such that the matrix of the morphism $\Omega_{Y, y} \rightarrow \mathcal{P}_{\mid Y, y}$ is

$$
\left(\begin{array}{cccccc}
1 & 0 & & \ldots & \ldots & 0 \\
0 & 1 & 0 & \ldots & \ldots & 0 \\
& \ldots & \ldots & \ldots & \ldots & \\
0 & \ldots & 1 & 0 & \ldots & 0 \\
0 & \ldots & 0 & f_{r} & \ldots & f_{n}
\end{array}\right) .
$$

That is, $\Omega_{Y, y} \simeq L^{\prime} \oplus L$ and $\mathcal{P}_{\mid Y, y} \simeq L^{\prime} \oplus \mathcal{O}$ and the morphism $\Omega_{Y}, y \rightarrow \mathcal{P}_{\mid Y, y}$ is $I d \oplus\left(f_{r}, \ldots, f_{n}\right)$.

Let $e_{1}, \ldots, e_{l}$ be a basis of $\left(\mathfrak{p} / \mathfrak{p}^{2}\right)_{y}=N$. As it was shown in the proof of Proposition 1.1, this basis induces a splitting of the exact sequence $0 \rightarrow \Omega_{Y} \rightarrow$ $\Omega_{N}[Y] \rightarrow \operatorname{End}_{Y}\left(\mathfrak{p} / \mathfrak{p}^{2}\right)^{*} \rightarrow 0 ;$ that is, one has a section $\operatorname{End}_{Y}\left(\mathfrak{p} / \mathfrak{p}^{2}\right)^{*} \rightarrow \Omega_{N}[Y]$ 
Let $\phi_{i j}$ be the basis of $\operatorname{End}_{Y}\left(\mathfrak{p} / \mathfrak{p}^{2}\right)^{*}$ induced by $e_{1}, \ldots, e_{l}$ and denote by $\frac{d e_{i}}{e_{j}}$ their image in $\Omega_{N}[Y]$ by such a section. Let $g_{i j} \in \mathcal{O}$ be the image of $\frac{d e_{i}}{e_{j}}$ by the morphism

$$
\Omega_{N}[Y]_{y} \rightarrow \mathcal{P}_{\mid Y, y} \simeq L^{\prime} \oplus \mathcal{O} \rightarrow \mathcal{O}
$$

and denote by $A$ the matrix with coefficients $g_{i j}$.

Theorem 4.3. With the preceding notations, assume that $S$ has codimension $n-$ $r+1$ and $C$ is monogenerated at every point. Denote by $c_{i}(A)$ the $i$-th coefficient of the characteristic polynomial of $A$. Then $\operatorname{Res}(\mathcal{P}, Q)$ is locally given by the Grothendieck symbol

$$
\left[\begin{array}{c}
Q\left(c_{1}(A), \ldots, c_{l}(A)\right) \omega_{r} \wedge \cdots \wedge \omega_{n} \\
f_{r} \ldots f_{n}
\end{array}\right]
$$

if $Q$ is a polynomial of degree $p=n-r+1$, and it is zero for $p>n-r+1$.

Proof. First of all, notice that $S$ is locally given by the zeros of $f_{r}, \ldots, f_{n}$, and since $S$ has codimension $n-r+1$, then $f_{r}, \ldots, f_{n}$ is a regular sequence. Let us consider the diagram (assume $Y=\operatorname{Spec} \mathcal{O}$, with $\mathcal{O}$ a local ring)

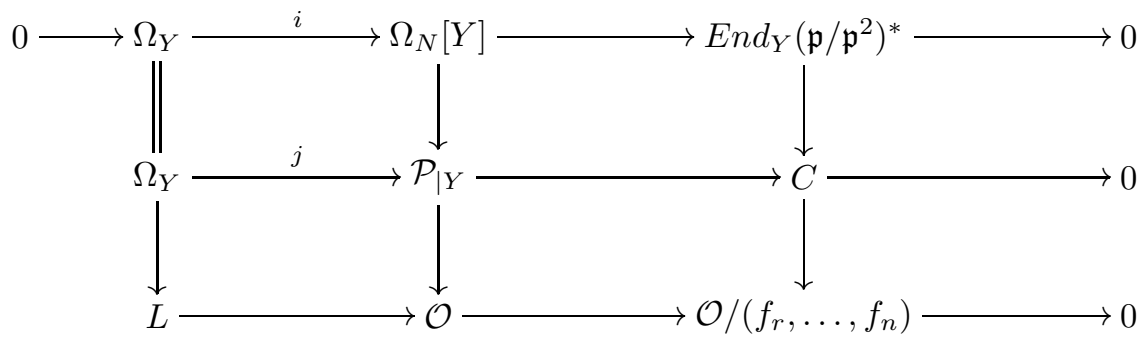

This diagram induces morphisms $\operatorname{Kos}_{\bullet}^{p}(i) \rightarrow \operatorname{Kos}_{\bullet}^{p}(j) \rightarrow \operatorname{Kos}_{\bullet}\left(f_{r}, \ldots, f_{n}\right)$, and this latter is a quasi-isomorphism, since $\operatorname{Kos}_{\bullet}(I d)$ is acyclic. Now, $\operatorname{Kos}_{\bullet}^{p}(i)$ is the complex $0 \rightarrow \Omega_{Y}^{p} \rightarrow \cdots \rightarrow S^{p} \operatorname{End}_{Y}\left(\mathfrak{p} / \mathfrak{p}^{2}\right)^{*} \rightarrow 0$ which induces a morphism $H^{0}\left(Y, S^{p} \operatorname{End}_{Y}\left(\mathfrak{p} / \mathfrak{p}^{2}\right)^{*}\right) \rightarrow H_{S}^{p}\left(Y, \Omega_{Y}^{p}\right)$ that takes $Q\left(s_{1}, \ldots, s_{l}\right)$ into $\operatorname{Res}_{S}(\mathcal{P}, Q)$.

On the other hand, Kos• $\left(f_{r}, \ldots, f_{n}\right)$ gives a morphism $H^{0}\left(S, \mathcal{O} /\left(f_{r}, \ldots, f_{n}\right)\right) \rightarrow$ $H_{S}^{p}\left(Y, \Omega_{Y}^{p}\right)(p=n-r+1)$ such that the image of 1 is $\left[\begin{array}{c}\omega_{r} \wedge \cdots \wedge \omega_{n} \\ f_{r} \ldots f_{n}\end{array}\right]$. Moreover, the morphisms

$$
S^{p} \operatorname{End}_{Y}\left(\mathfrak{p} / \mathfrak{p}^{2}\right)^{*} \rightarrow S^{p} C \rightarrow S^{p}\left(\mathcal{O} /\left(f_{r}, \ldots, f_{n}\right)\right) \simeq \mathcal{O} /\left(f_{r}, \ldots, f_{n}\right)
$$

take $Q\left(s_{1}, \ldots, s_{l}\right)$ into $Q\left(c_{1}(A), \ldots, c_{l}(A)\right)$. Taking it all together, the result follows. If $p>n-r+1, H^{p}\left(\operatorname{Kos}_{\bullet}\left(f_{r}, \ldots, f_{n}\right)\right)=0$ and one concludes.

Case of a vector field. Let $\mathfrak{D}$ be a Pfaff system of rank $1, r=1$, which we shall call a vector field (in other words, a one-dimensional foliation). As before, $j: Y \hookrightarrow X$ is a closed subscheme which is the solution of $\mathfrak{D}$ and is defined by the sheaf of ideals $\mathfrak{p}$. We again denote $S=\operatorname{Sin}\left(\mathfrak{D}_{\mid Y}\right) \cap \operatorname{Sin}(Y)$.

Theorem 4.4. Assume that $\mathfrak{p} / \mathfrak{p}^{2}$ is a coherent locally free module of rank $l$. Let $S_{\alpha}$ be the connected components of $S$ and $Q \in H^{0}\left(Y, \mathcal{O}_{Y}\right)\left[s_{1}, \ldots, s_{l}\right]$ a homogeneous polynomial of degree $d=\operatorname{dim} Y$. For each $S_{\alpha}$ there exists an element in $H_{S_{\alpha}}^{d}\left(Y, \Omega_{Y}^{d}\right)$, that we denote by $\operatorname{Res}_{S_{\alpha}}(\mathfrak{D}, Q)$, such that

$$
\sum_{\alpha} \phi_{\alpha}\left(\operatorname{Res}_{S_{\alpha}}(\mathfrak{D}, Q)\right)=Q\left(c_{1}\left(\mathfrak{p} / \mathfrak{p}^{2}\right), \ldots, c_{l}\left(\mathfrak{p} / \mathfrak{p}^{2}\right)\right)
$$


where $\phi_{\alpha}: H_{S_{\alpha}}^{d}\left(Y, \Omega_{Y}^{d}\right) \rightarrow H^{d}\left(Y, \Omega_{Y}^{d}\right)$ is the natural morphism.

Remarks. 1) If $Y$ is a proper variety over a field $k$, one has a canonical morphism

$$
H^{d}\left(Y, \Omega_{Y}^{d}\right) \rightarrow k
$$

which together with the natural morphism $H_{S_{\alpha}}^{d}\left(Y, \Omega_{Y}^{d}\right) \rightarrow H^{d}\left(Y, \Omega_{Y}^{d}\right)$ gives a morphism $H_{S_{\alpha}}^{d}\left(Y, \Omega_{Y}^{d}\right) \rightarrow k$. Therefore the residues $\operatorname{Res}_{S_{\alpha}}(\mathfrak{D}, Q)$ may be considered as numbers (taking their image into $k$ under that morphism). The formula $(*)$ gives us the global sum of these numbers along the subvariety $Y$.

2) If $Y$ is a variety over a field $k$ and $S_{\alpha}$ are closed points, one has canonical morphisms

$$
H_{S_{\alpha}}^{d}\left(Y, \Omega_{Y}^{d}\right) \rightarrow k
$$

and therefore the residues $\operatorname{Res}_{S_{\alpha}}(\mathfrak{D}, Q)$ may be understood as numbers. If in addition $Y$ is a proper variety, the morphism $H_{S_{\alpha}}^{d}\left(Y, \Omega_{Y}^{d}\right) \rightarrow k$ coincides with the composition of the canonical morphisms $H_{S_{\alpha}}^{d}\left(Y, \Omega_{Y}^{d}\right) \rightarrow H^{d}\left(Y, \Omega_{Y}^{d}\right)$ and $H^{d}\left(Y, \Omega_{Y}^{d}\right) \stackrel{\sim}{\rightarrow} k$, so the formula $(*)$ gives the global sum of these numbers.

Explicit computation (for a vector field). Let us assume that $Y$ is a smooth variety over a field $k$ and that $S_{\alpha}$ is a closed point, denoted by $p$. Let $x_{1}, \ldots, x_{d}$ be a system of parameters at $p$ and $e_{1}, \ldots, e_{l}$ a basis of $\mathfrak{p} / \mathfrak{p}^{2}$ at $p$. Let $D$ be a local generator of $\mathfrak{D}^{*} \hookrightarrow T_{X}$ at $p$. Let

$$
\bar{D}=f_{1} \frac{\partial}{\partial x_{1}}+\cdots+f_{d} \frac{\partial}{\partial x_{d}}
$$

be the local generator of $\mathfrak{D}_{\mid Y}^{*} \hookrightarrow T_{Y}$ at $p$ induced by $D$, and let $L_{p}$ be the endomorphism induced in $\left(\mathfrak{p} / \mathfrak{p}^{2}\right)_{p}$. Denote $c_{1}\left(L_{p}\right), \ldots, c_{l}\left(L_{p}\right)$ the coefficients of the characteristic polynomial of $L_{p}$.

Theorem 4.5. With the notations above, one has that

$$
\operatorname{Res}_{p}(\mathfrak{D}, Q)=\operatorname{Res}_{p}\left(\frac{Q\left(c_{1}\left(L_{p}\right), \ldots, c_{l}\left(L_{p}\right)\right) \cdot \mathrm{d} x_{1} \wedge \cdots \wedge \mathrm{d} x_{d}}{f_{1} \ldots f_{d}}\right) .
$$

(In the right side, $\operatorname{Res}_{p}$ denotes the ordinary residue of a differential form.)

Proof. It follows from 4.3, taking into account that under the canonical morphism $H_{p}^{d}\left(Y, \Omega_{Y}^{d}\right) \rightarrow k$, the Grothendieck symbol

$$
\left[\begin{array}{c}
Q\left(c_{1}\left(L_{p}\right), \ldots, c_{l}\left(L_{p}\right)\right) \cdot \mathrm{d} x_{1} \wedge \cdots \wedge \mathrm{d} x_{d} \\
f_{1} \ldots f_{d}
\end{array}\right]
$$

takes into $\operatorname{Res}_{p}\left(\frac{Q\left(c_{1}\left(L_{p}\right), \ldots, c_{l}\left(L_{p}\right)\right) \cdot \mathrm{d} x_{1} \wedge \cdots \wedge \mathrm{d} x_{d}}{f_{1} \cdots f_{d}}\right)$.

Case of a blowing up. Let $Z$ be an irreducible component of codimension $d$ of the singular locus of a vector field $\mathfrak{D}$ on $X$. Let $p$ be the generic point of $Z$, $X_{p}=\operatorname{Spec} \mathcal{O}_{p}$ and $X^{\prime} \rightarrow X_{p}$ the blowing up at $p$. Let $E$ be the exceptional fibre, which is a projective space of dimension $d-1$ over $k(p)=$ field of meromorphic functions of $Z$. The vector field in $X$ induces another in $X^{\prime}$, that we denote $\mathfrak{D}^{\prime}$. Locally this is given as follows:

Let $x_{1}, \ldots, x_{n}$ be local parameters at a closed nonsingular point of $Z$, such that $Z=\left\{x_{1}=\cdots=x_{d}=0\right\}$. Let

$$
f_{1} \frac{\partial}{\partial x_{1}}+\cdots+f_{n} \frac{\partial}{\partial x_{n}}
$$


be a local generator of the vector field. The multiplicity of the vector field at $p$ is the minimum of the multiplicities of the $f_{i}$ at $p$. We shall denote it by $\mu$.

Let us take $x_{1}$ as a blowing up parameter

$$
\begin{aligned}
x_{1}^{\prime} & =x_{1}, \\
x_{i}^{\prime} & =\frac{x_{i}}{x_{1}}, \quad i=2, \ldots, d, \\
x_{j}^{\prime} & =x_{j}, \quad j=d+1, \ldots, n .
\end{aligned}
$$

Then

$$
\begin{aligned}
& \frac{\partial}{\partial x_{1}}=\frac{\partial}{\partial x_{1}^{\prime}}-\sum_{i=2}^{d} \frac{x_{i}^{\prime}}{x_{1}^{\prime}} \frac{\partial}{\partial x_{i}^{\prime}}, \\
& \frac{\partial}{\partial x_{i}}=\frac{1}{x_{1}^{\prime}} \frac{\partial}{\partial x_{i}^{\prime}}, \quad i=2, \ldots, d, \\
& \frac{\partial}{\partial x_{j}}=\frac{\partial}{\partial x_{j}^{\prime}}, \quad j=d+1, \ldots, n .
\end{aligned}
$$

Then, if we denote

$$
f_{i}^{\prime}=\frac{f_{i}}{x_{1}^{\mu}}, \quad(\mu=\text { multiplicity of } \mathfrak{D} \text { at } p)
$$

one has that

$$
f_{1} \frac{\partial}{\partial x_{1}}+\cdots+f_{n} \frac{\partial}{\partial x_{n}}=x_{1}^{\prime \mu-1}\left(f_{1}^{\prime} x_{1}^{\prime} \frac{\partial}{\partial x_{1}^{\prime}}+\sum_{i=2}^{d}\left(f_{i}^{\prime}-x_{i}^{\prime} f_{1}^{\prime}\right) \frac{\partial}{\partial x_{i}^{\prime}}+\sum_{j=d+1}^{n} f_{j}^{\prime} x_{1}^{\prime} \frac{\partial}{\partial x_{j}^{\prime}}\right) .
$$

$Z$ is said to be nondicritical if $\left(f_{2}^{\prime}-x_{2}^{\prime} f_{1}^{\prime}, \ldots, f_{d}^{\prime}-x_{d}^{\prime} f_{1}^{\prime}\right) \nsubseteq\left(x_{1}^{\prime}\right)$. Then a local generator of $\left(\mathfrak{D}^{\prime}\right)^{*}$ is

$$
\begin{gathered}
f_{1}^{\prime} x_{1}^{\prime} \frac{\partial}{\partial x_{1}^{\prime}}+\sum_{i=2}^{d}\left(f_{i}^{\prime}-x_{i}^{\prime} f_{1}^{\prime}\right) \frac{\partial}{\partial x_{i}^{\prime}}+\sum_{j=d+1}^{n} f_{j}^{\prime} x_{1}^{\prime} \frac{\partial}{\partial x_{j}^{\prime}} \quad \text { if } Z \text { is nondicritical }, \\
f_{1}^{\prime} \frac{\partial}{\partial x_{1}^{\prime}}+\sum_{i=2}^{d} \frac{\left(f_{i}^{\prime}-x_{i}^{\prime} f_{1}^{\prime}\right)}{x_{1}^{\prime}} \frac{\partial}{\partial x_{i}^{\prime}}+\sum_{j=d+1}^{n} f_{j}^{\prime} \frac{\partial}{\partial x_{j}^{\prime}} \quad \text { if } Z \text { is dicritical, }
\end{gathered}
$$

which is called proper transform of the vector field. It is easy to see that $Z$ is nondicritical if and only if $\mathfrak{D}_{\mid E}^{\prime}{ }^{*} \hookrightarrow T_{E / k(p)}$.

Suppose that $p$ is a nondicritical singularity of $\mathfrak{D}$. Then $\mathfrak{D}^{\prime}$ induces a vector field $\mathfrak{D}^{\prime \prime}$ on the normal bundle of $E$ over $X^{\prime}, \mathbf{N}_{E / X^{\prime}}$. This vector field is trivial over $k(p)$, that is, it is locally given by a derivation which vanishes over $k(p)$. Moreover $E$ is the solution of $\mathfrak{D}^{\prime \prime}$, considering $E$ as a subvariety of $\mathbf{N}_{E / X^{\prime}}$ via the null section. In addition, the restriction of $\mathfrak{D}^{\prime \prime}$ to $E$ coincides with the restriction of $\mathfrak{D}^{\prime}$.

We can apply the residue theory to the Pfaff system $\mathfrak{D}^{\prime \prime}$, taking $\mathbf{N}_{E / X^{\prime}}$ as the ambient variety and $E$ as the solution subvariety (and considering both of them as varieties over $k(p))$.

All the polynomials of one variable of degree $d-1$ are of the form $\lambda \cdot x^{d-1}$, with $\lambda \in k(p)$. We shall only consider the polynomial $Q=(-x)^{d-1}$. Then Theorems 4.4 and 4.5 stand as follows: 
Theorem 4.6. Let $q_{i}$ be the generic points of the singular locus of $\mathfrak{D}^{\prime}$. For each $q_{i}$ there exists a "number" in $k(p)$, denoted by $\operatorname{Res}_{q_{i}}(\mathfrak{D}, E)$, such that

$$
\sum \operatorname{Res}_{q_{i}}(\mathfrak{D}, E)=c_{1}\left(N_{E / X^{\prime}}\right)^{d-1}=E^{d}=(-1)^{d-1}
$$

via the canonical isomorphism $H^{d-1}\left(E, \Omega_{E / k(p)}^{d-1}\right) \stackrel{\sim}{\rightarrow} k(p)$.

Moreover, if $q_{i}$ has the same codimension as $p$, then

$$
\operatorname{Res}_{q_{i}}(\mathfrak{D}, E)=\operatorname{Res}_{q_{i}}\left(\frac{g^{d-1} \cdot d x_{2} \wedge \cdots \wedge d x_{d}}{f_{2} \cdots f_{d}}\right)
$$

where $g=$ homothety induced by the vector field in $\left(N_{E / X^{\prime}}\right)_{q_{i}}$ and

$$
f_{2} \frac{\partial}{\partial x_{2}}+\cdots+f_{d} \frac{\partial}{\partial x_{d}}
$$

is a local generator of $\left(\mathfrak{D}^{\prime}\right)_{\mid E}^{*} \hookrightarrow T_{E / k(p)}$ at $q_{i}\left(x_{2}, \ldots, x_{d}\right.$ is a local system of parameters at $q_{i}$, considering $E$ as a variety over $\left.k(p)\right)$.

Now we see the relation between the residue and the linear part of the vector field, for singularities of codimension 2 .

Let $p$ be a singular point of a vector field $\mathfrak{D}, \mathcal{O}_{p}$ the local ring at $p$ and $\mathfrak{m}$ the maximal ideal. If $D$ is a local generator of $\mathfrak{D}^{*}$ at $p$, it induces an endomorphism

$$
\begin{aligned}
L_{p}: \mathfrak{m} / \mathfrak{m}^{2} & \rightarrow \mathfrak{m} / \mathfrak{m}^{2} \\
\bar{f} & \mapsto \overline{D f}
\end{aligned}
$$

which is not zero if and only if $\mathfrak{D}$ has multiplicity 1 at $p$. We say that $L_{p}$ is the linear part of the vector field at $p$.

Corollary 4.7. Let $p$ be a singularity of codimension 2 and nondicritical and let $X_{p}=\operatorname{Spec} \mathcal{O}_{p}$. Let $X^{\prime} \rightarrow X$ be the blowing up at $p$, with exceptional fibre $E$.

1) $\sum \operatorname{Res}_{q_{i}}(\mathfrak{D}, E)=-1$. Therefore there exist some $q_{i}$ such that $\operatorname{Res}_{q_{i}}(\mathfrak{D}, E) \notin$ $\mathbb{Q}^{+} \cup 0$ (in zero characteristic).

2) If $L_{q_{i}}=\left(\begin{array}{ll}\lambda & \gamma \\ 0 & \mu\end{array}\right)$ is the linear part of the vector field at $q_{i}$, where $\mu \neq 0$ and $\lambda$ is the eigenvalue corresponding to the exceptional fibre, then

$$
\operatorname{Res}_{q_{i}}(\mathfrak{D}, E)=\operatorname{Tr}\left(\frac{\lambda}{\mu}\right)
$$

where $\operatorname{Tr}: k\left(q_{i}\right) \rightarrow k(p)$ is the trace morphism (which is the identity if $q_{i}$ is rational over $p$ ).

3) Let $q \in E$ be a nondicritical singular point and $X^{\prime \prime} \rightarrow X^{\prime}$ the blowing up at $q$ with exceptional fibre $E^{\prime \prime}$. If $E^{\prime}$ denotes the proper transform of $E$ and $q^{\prime}=E^{\prime} \cap E^{\prime \prime}$, then

$$
\operatorname{Res}_{q^{\prime}}\left(\mathfrak{D}, E^{\prime}\right)=\operatorname{Res}_{q}(\mathfrak{D}, E)-1 .
$$

Proof. 1) and 2) follow from Theorem 4.6. 3) follows from Theorem 4.6 and from the equality

$$
E^{2}=\left(E^{\prime}+E^{\prime \prime}\right)^{2}=E^{\prime 2}+2 E^{\prime} \cdot E^{\prime \prime}+E^{\prime \prime 2}=E^{\prime 2}+2-1=E^{\prime 2}+1
$$

This corollary generalizes the properties of the residue of Camacho and Sad on surfaces for singularities of codimension 2 . 
A computation in the singular case. Let $\mathfrak{D}$ be a vector field on an integral surface $X$ (possibly singular) and with an invariant curve $C$ (possibly singular too). Assume $C$ is integral and a locally complete intersection in $X$. Let $\Sigma$ be the field of functions of $C$ and let us denote $S=\operatorname{Sin}(C) \cap \operatorname{Sin}\left(\mathfrak{D}_{\mid C}\right)$. Let $p \in S$. Then $\operatorname{Res}_{p}(\mathfrak{D}, C) \in H_{p}^{1}\left(C, \Omega_{C}\right)$. It is easy to see that $H_{p}^{1}\left(C, \Omega_{C}\right)$ may be identified with $\Omega_{\Sigma / k} / \Omega_{\mathcal{O} / k}$, where $\mathcal{O}$ is the local ring of $C$ at $p$. Given $\omega \in \Omega_{\Sigma / k}$, we shall denote by $[\omega]$ its class in $H_{p}^{1}\left(C, \Omega_{C}\right)$. One has a morphism $\operatorname{Res}_{p}: H_{p}^{1}\left(C, \Omega_{C}\right) \rightarrow k$, such that $\operatorname{Res}_{p}([\omega])$ is the ordinary residue at $p$ of the 1 -form $\omega$ (this is the "ordinary" residue for singular varieties).

Let $p \in S$, and let $f=0$ be a local (at $p$ ) equation of $C$ in $S$. Let $D$ be a local generator of $\mathfrak{D}$ at $p$. Then $D f=g \cdot f$. Let $\omega \neq 0$ be a regular 1 -form of $\mathcal{O}$, that is, $\omega \in \Omega_{\mathcal{O} / k}$. Then

Theorem 4.8. With the preceding notations,

$$
\operatorname{Res}_{p}(\mathfrak{D}, C)=\left[\frac{g_{\mid C} \cdot \omega}{\omega\left(D_{\mid C}\right)}\right] .
$$

Then, as a number (via the morphism $\operatorname{Res}_{p}: H_{p}^{1}\left(C, \Omega_{C}\right) \rightarrow k$ ),

$$
\operatorname{Res}_{p}(\mathfrak{D}, C)=\operatorname{Res}_{p}\left[\frac{g_{\mid C} \cdot \omega}{\omega\left(D_{\mid C}\right)}\right] .
$$

This residue may be computed in two ways: 1) In terms of the desingularization of the curve: If $\pi: C^{\prime} \rightarrow C$ is the desingularization of $C$, then

$$
\operatorname{Res}_{p}\left[\frac{g_{\mid C} \cdot \omega}{\omega\left(D_{\mid C}\right)}\right]=\sum_{\pi(q)=p} \operatorname{Res}_{q}\left[\frac{g_{\mid C} \cdot \omega}{\omega\left(D_{\mid C}\right)}\right] .
$$

2) This residue may be expressed as a Grothendieck symbol, and this one may be computed as a Grothendieck symbol on the surface:

$$
\operatorname{Res}_{p}\left[\frac{g_{\mid C} \cdot \omega}{\omega\left(D_{\mid C}\right)}\right]=\left[\begin{array}{c}
g_{\mid C} \cdot \omega \\
\omega\left(D_{\mid C}\right)
\end{array}\right]=\left[\begin{array}{c}
g \cdot \omega^{\prime} \wedge \mathrm{d} f \\
\omega^{\prime}(D), f
\end{array}\right]
$$

where $\omega^{\prime}$ is a regular form in $X$ such that $\omega_{\mid C}^{\prime}=\omega$.

Proof. Let us consider the extension of the logarithmic differentials

$$
0 \rightarrow \Omega_{C} \rightarrow \Omega_{N}[C] \rightarrow \mathcal{O}_{C} \rightarrow 0
$$

where $N$ is the conormal module of $C$ over $X$. If $j: C \hookrightarrow X$ is the natural immersion, then $\Omega_{N}[C]=j^{*} \Omega_{X}[\log C]$. Let $\mathcal{O}$ be the local ring at $p$, and $\mathcal{E}=\Omega_{N}[C]_{p}$. One has that $\mathcal{E}=\Omega_{\mathcal{O}} \oplus \mathcal{O} \cdot \frac{\mathrm{d} f}{f}$. The vector field $\mathfrak{D}$ gives a morphism $\phi: \mathcal{E} \rightarrow \mathcal{O}$, which maps $\omega+\lambda \frac{\mathrm{d} f}{f}$ into $\omega\left(D_{\mid C}\right)+\lambda g_{\mid C}$ (with the preceding notations). Let us denote $\bar{\Omega}_{\mathcal{O}}$ the quotient of $\Omega_{\mathcal{O}}$ by its torsion submodule. We have a commutative diagram

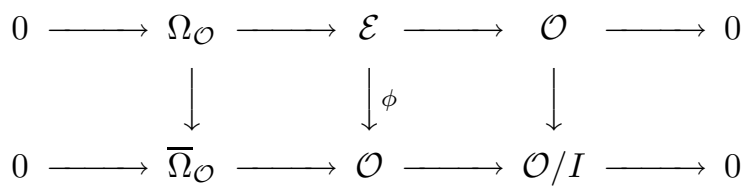


where $I$ is the ideal that defines the singular locus of $\mathfrak{D}_{\mid C}$ at $p$. Then one has a morphism

$$
\mathcal{O} / I \rightarrow H_{p}^{1}\left(\bar{\Omega}_{\mathcal{O}}\right) \stackrel{\sim}{\longleftarrow} H_{p}^{1}\left(\Omega_{\mathcal{O}}\right)
$$

By construction, $\operatorname{Res}_{p}(\mathfrak{D}, C)$ is the image of 1 under this morphism. But, via the identification of $H_{p}^{1}\left(\bar{\Omega}_{\mathcal{O}}\right)$ with $\Omega_{\Sigma} / \Omega_{\mathcal{O}}$, and $H_{p}^{1}(\mathcal{O})$ with $\Sigma / \mathcal{O}$, one has that $\mathcal{O} / I$ is isomorphic to the kernel of the morphism $\Omega_{\Sigma} / \Omega_{\mathcal{O}} \rightarrow \Sigma / \mathcal{O}$, and the explicit isomorphism is given by the snake lemma applied to the diagram

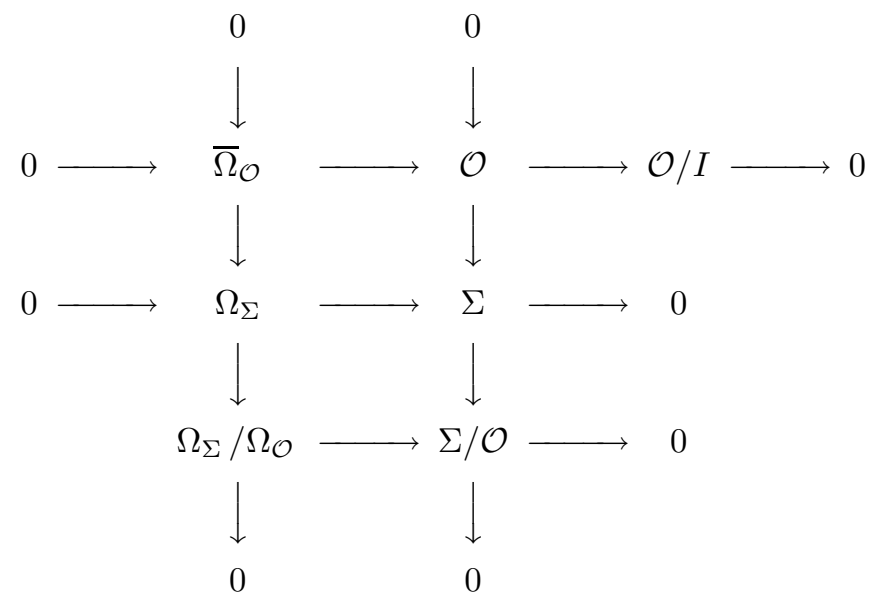

This explicit isomorphism takes 1 into $\left[\frac{g_{\mid C} \cdot \omega}{\omega\left(D_{\mid C}\right)}\right]$, so one concludes. The computations 1) and 2) follow from the general theory of ordinary residues and Grothendieck symbols.

\section{REFERENCES}

[1] M. F. Atiyah: Complex analytic connections in fibre bundles. Trans. Amer. Math. Soc. 85 (1957), pp. 181-207. MR 19:172c

[2] C. Camacho and P. Sad: Invariant varieties through singularities of holomorphic vector fields. Annals of Math. 115 (1982), pp. 579-595. MR 83m:58062

[3] B. Gmira: Une généralisation d'un théorème de C. Camacho et P. Sad relatif aus feuilletages holomorphes singuliers. Thèse de III ${ }^{\mathrm{ème}}$ cycle, Lille (1984).

[4] A. Grothendieck: Classes de Chern et représentations linéaires des groupes discrets. Dix esposés sur la cohomologie des schémas. North-Holland, (1968), pp. 215-305. MR 42:280

[5] D. Lehmann and T. Suwa: Residues of holomorphic vector fields relative to singular invariant subvarieties. J. of Differential Geom. 42, 1995, 165-192. MR 96f:32064

[6] D. Lehmann: Résidus des sous-variétés invariantes d'un feuilletage singulier. Ann. Inst. Fourier 41 (1991) pp. 211-258 (Bucarest). MR 92k:57054

[7] A. Lins Neto: Algebraic solutions of polynomial differential equations and foliations in dimension two. Holomorphic Dynamics, Mexico 1986, Lecture Notes in Math. 1345 (1988) pp. 192-232. MR 90c:58142

[8] A. Lins Neto: Complex codimension one foliations leaving a compact submanifold invariant. Dynamical Systems and Bifurcation Theory 1985, Pitman Research Notes in Mathematics Series 160, Longman Scientific and Technical, Harlow, New York (1987), pp. 295-317. MR 88m:57036

[9] M. Soares: A note on algebraic solutions of foliations in dimension 2. Dynamical Systems 1990, Pitman Research Notes in Mathematics Series 285, Longman Scientific and Technical, Harlow, New York (1993), pp. 250-254. MR 94k:32054

[10] T. Suwa: Indices of holomorphic vector fields relative to invariant curves on surfaces. Proc. Amer. Math. Soc. 123, 1995, 2989-2997. MR 95m:32054 
[11] T. Suwa: Residues of complex analytic foliation singularities. J. Math. Soc. Japan, 36, (1984) pp. 37-45. MR 85f:32016

[12] T. Suwa: Residues of complex analytic foliations relative to singular invariant subvarieties. Proceedings of the International Seminar on Complex Geometry and Singularities, Beijing (1994) pp. 230-245. MR 98i:32053

Departamento de Matemáticas, Universidad de Salamanca, Plaza de la Merced 1-4, 37008 Salamanca, Spain

E-mail address: fsancho@gugu.usal.es 